\title{
Optical solutions and other solutions for Radhakrishnan-Kundu-Laksmannan equation by using improved modified extended tanh-function method
}

\author{
Ahmed M. Elsherbeny ${ }^{1}$, Reda El-Barkouky ${ }^{1}$, Hamdy M. Ahmed ${ }^{2}$, \\ Rabab M. I. El-Hassani ${ }^{1}$, Ahmed H. Arnous ${ }^{2}$ \\ ${ }^{1}$ Department of Physics and Mathematics Engineering, Faculty of Engineering, \\ Ain Shams University, Cairo, Egypt. \\ 2 Department of Physics and Engineering Mathematics, Higher Institute of Engineering, \\ El-Shorouk Academy, El-Shorouk City, Cairo, Egypt.
}

\begin{abstract}
This paper studies Radhakrishnan-Kundu-Laksmannan equation which is used to describe the pulse propagation in optical fiber communications. By using improved modified extended tanh-function method various types of solutions are extracted such as bright solitons, singular solitons, singular periodic wave solutions, Jacobi elliptic solutions, periodic wave solutions and Weierstrass elliptic doubly periodic solutions. Moreover, some of the obtained solutions are represented graphically.
\end{abstract}

Keywords: Radhakrishnan-Kundu-Laksmannan, Solitons, Jacobi elliptic solutions, Improved modified extended tanh-function method.

\section{Introduction}

Nonlinear evolution equations play a major role in a variety of scientific and engineering fields, such as ocean engineering, optical fiber communications, plasma physics and fluid dynamics. The studies of Soliton solutions for non-linear evolution equation attracted many researchers and one can review the articles ([1]-[27]). The Radhakrishnan-Kundu-Laksmannan (RKL) equation that describe the pulse propagation in optical fibers has been studied by some authors. In [1], author obtained a single soliton solution for the RKL equation. In [2], authors studied bifurcations of exact travelling wave solutions for the generalized RKL equation. In [3], author obtained bright and dark soliton solutions for the Radhakrishnan-KunduLakshmanan equation by Lie group analysis. In [4], authors established optical solitons of the RKE equation by the extended trial function integration scheme. In [5], authors obtained chrip-free optical bight soliton solutions for the RKE equation. In [7], author obtained periodic and solitary wave forms of exact general solutions for the RKE equation.

In this work, the improved modified extended tanh-function method is implemented to the RadhakrishnanKundu-Laksmannan equation. The proposed method gives more and variety types of solutions than other methods. These solutions including bright solitons, singular solitons, singular periodic wave solutions, Jacobi elliptic solutions, periodic wave solutions and Weierstrass elliptic doubly periodic solutions. In the end of the paper, two-dimensional and three-dimensional graphs of some solutions are introduced for knowing the physical interpretation. 


\section{Governing model}

The RKL equation is given by [7]:

$$
i q_{t}+a q_{x x}+b|q|^{2 n} q+i \beta q_{x x x}+i \alpha\left(|q|^{2 n} q\right)_{x}=0 ; \quad n \neq 0 .
$$

where $q=q(x, t)$ is a dependant complex-valued variable represents the wave profile, the temporal and spatial coordinates are represented by the variables $t$ and $x$ respectively, $n \in Q$ is the arbitary index, $i^{2}=-1, a, b$, $\alpha$ and $\beta$ are parameters of equation (1).

Assuming that the solution of equation (1) has the form:

$$
q(x, t)=P(\zeta) e^{i\left(-k x+\omega t+\theta_{0}\right)}, \zeta=x-\nu t
$$

Where $k, w$ and $\theta_{0}$ represent the soliton frequency, the wave number and the phase constant. Substituting by equation (2) into equation (1), the real part can be written as:

$$
-P(\zeta)\left(a k^{2}+\beta k^{3}+\omega\right)+(a+3 \beta k) P^{\prime \prime}(\zeta)+(b+\alpha k) P(\zeta)^{2 n+1}=0
$$

And the imaginary part can be written as:

$$
-P^{\prime}(\zeta)\left(2 a k+3 \beta k^{2}+\nu\right)+\alpha(2 n+1) P(\zeta)^{2 n} P^{\prime}(\zeta)+\beta P^{(3)}(\zeta)=0
$$

Integrating the imaginary part (4) with respect to $\zeta$, setting the integration constant to zero, so equation (4) can be rewritten as:

$$
-P(\zeta)\left(2 a k+3 \beta k^{2}+\nu\right)+\alpha P(\zeta)^{2 n+1}+\beta P^{\prime \prime}(\zeta)=0
$$

From equation (3), and considering the principle of linear independence, we can conclude:

$$
k=-\frac{a}{3 \beta} \text { and } \omega=-\frac{2 a^{3}}{27 \beta^{2}}, \text { where } a=\frac{3 \beta b}{\alpha}
$$

Taking into consideration equation (6), and applying the transformation $P=Q^{\frac{1}{n}}$, equation (5) can be simply rewritten as:

$$
-a^{2} n^{2} Q(\zeta)^{2}-3 \alpha \beta n^{2} Q(\zeta)^{4}+3 \beta \nu n^{2} Q(\zeta)^{2}-3 \beta^{2} n Q(\zeta) Q^{\prime \prime}(\zeta)+3 \beta^{2} n Q^{\prime}(\zeta)^{2}-3 \beta^{2} Q^{\prime}(\zeta)^{2}=0
$$

Applying the integration scheme over equation(7) leads to the solution of equation (1) as shown in the next sections.

\section{Improved modified extended tanh-function method}

In this section, the improved modified extended tanh-function method is described as follows [1, 28]:

Assuming the non-linear evolution equation (NLEE) with two independent variables $t$ and $x$ :

$$
P\left(u, u_{x}, u_{t}, u_{x x}, u_{t t}, u_{x t}, \ldots\right)=0
$$


where $u=u(x, t)$ is an unknown function symbolize the solution of the NLEE (8) and $P$ is a polynomial in $u$ and its various partial derivatives. Mostly, the soliton solution integration schemes principally based on supposing a variable $\xi$ as a linear conjunction of the independent variables $x$ and $t$ to convert the NLEE into an ordinary differential equation(ODE):

$$
H\left(S, S^{\prime}, S^{\prime \prime}, S^{\prime \prime \prime}, \ldots\right)=0
$$

The elaborated procedures of the proposed integration scheme will be presented in the following steps:

Step-1: Considering that the NLEE (8) holds

$$
u(x, t)=u(\xi), \xi=x-\nu t .
$$

where $\nu$ is a constant symbolizes the wave speed and will be determined later. Employing the transformation of equation (10) will leads to the ODE (9).

Step-2: Supposing that the solution of equation (9) has the form:

$$
u(\xi)=\sum_{i=0}^{N} \alpha_{i} \phi(\xi)^{i}+\sum_{j=1}^{N} \beta_{j} \phi(\xi)^{j} .
$$

where $\phi(\xi)$ holds

$$
\phi^{\prime}(\xi)=\epsilon \sqrt{a_{0}+a_{1} \phi(\xi)+a_{2} \phi(\xi)^{2}+a_{3} \phi(\xi)^{3}+a_{4} \phi(\xi)^{4}} .
$$

where $\epsilon= \pm 1$. By virtue of this, different kinds of basic solutions can be revealed.

Step-3: The non-negative integer $N$ is be identified in equation (11) by clenching the balance principle between the non-linear term with greatest order derivative term in equation (9).

Step-4: Taking into account (12), and substituting by equation (11) into (9), a polynomial of $\phi(\xi)$ is recovered. By equating the coefficients of $\phi^{i}(\xi)$ to zero, an overdetermined system is emerged. Thus, the real constants $\nu, \alpha_{i}(i=0,1, \ldots, N), \beta_{i}(i=0,1, \ldots, N)$ are recovered by solving the acquired overdetermined system. Consequently, the soliton solution of equation (1) will be obtained.

\section{Solitons and other solutions For the RKL equation}

The solution of equation (1) can be written as $q(x, t)=P(x-\nu t) e^{i\left(-k x+\omega t+\theta_{0}\right)}$, where $P=Q^{\frac{1}{n}}$ and this leads to equation (7). By applying the homogeneous balance principle, the solution of equation (7) can be written as:

$$
Q(\xi)=\alpha_{0}+\alpha_{1} \phi(\xi)+\frac{\beta_{1}}{\phi(\xi)} .
$$

Substituting by equation (13) in equation (7), yields to a polynomial of $\phi(\xi)$. Equating the coefficients of this polynomial to zero, leads to the following system of algebraic equation as follow:

For $\phi(\xi)^{-4}$ :

$$
-3 a_{0} \beta^{2} \beta_{1}^{2}(n-1)+6 a_{0} \beta^{2} \beta_{1}^{2} n+3 \alpha \beta \beta_{1}^{4} n^{2}=0 .
$$


For $\phi(\xi)^{-3}$ :

$$
6 a_{0} \alpha_{0} \beta^{2} \beta_{1} n-3 a_{1} \beta^{2} \beta_{1}^{2}(n-1)+\frac{9}{2} a_{1} \beta^{2} \beta_{1}^{2} n+12 \alpha \alpha_{0} \beta \beta_{1}^{3} n^{2}=0 .
$$

For $\phi(\xi)^{-2}$ :

$$
\begin{aligned}
& a^{2} \beta_{1}^{2} n^{2}+\frac{9}{2} a_{1} \alpha_{0} \beta^{2} \beta_{1} n+6 a_{0} \alpha_{1} \beta^{2} \beta_{1}(n-1)+6 a_{0} \alpha_{1} \beta^{2} \beta_{1} n-3 a_{2} \beta^{2} \beta_{1}^{2}(n-1) \\
& +3 a_{2} \beta^{2} \beta_{1}^{2} n+12 \alpha \alpha_{1} \beta \beta_{1}^{3} n^{2}+18 \alpha \alpha_{0}^{2} \beta \beta_{1}^{2} n^{2}-3 \beta \beta_{1}^{2} \nu n^{2}=0 .
\end{aligned}
$$

For $\phi(\xi)^{-1}$ :

$$
\begin{aligned}
& 2 a^{2} \alpha_{0} \beta_{1} n^{2}+3 a_{2} \alpha_{0} \beta^{2} \beta_{1} n+6 a_{1} \alpha_{1} \beta^{2} \beta_{1}(n-1)+6 a_{1} \alpha_{1} \beta^{2} \beta_{1} n-3 a_{3} \beta^{2} \beta_{1}^{2}(n-1) \\
& +\frac{3}{2} a_{3} \beta^{2} \beta_{1}^{2} n-6 \alpha_{0} \beta \beta_{1} \nu n^{2}+12 \alpha \alpha_{0}^{3} \beta \beta_{1} n^{2}+36 \alpha \alpha_{1} \alpha_{0} \beta \beta_{1}^{2} n^{2}=0 .
\end{aligned}
$$

For $\phi(\xi)^{0}$ :

$$
\begin{aligned}
& 2 a^{2} \alpha_{1} \beta_{1} n^{2}+a^{2} \alpha_{0}^{2} n^{2}+\frac{3}{2} a_{1} \alpha_{1} \alpha_{0} \beta^{2} n+\frac{3}{2} a_{3} \alpha_{0} \beta^{2} \beta_{1} n-3 a_{0} \alpha_{1}^{2} \beta^{2}(n-1) \\
& +6 a_{2} \alpha_{1} \beta^{2} \beta_{1}(n-1)+6 a_{2} \alpha_{1} \beta^{2} \beta_{1} n-3 a_{4} \beta^{2} \beta_{1}^{2}(n-1)-3 \alpha_{0}^{2} \beta \nu n^{2}-6 \alpha_{1} \beta \beta_{1} \nu n^{2} \\
& +3 \alpha \alpha_{0}^{4} \beta n^{2}+36 \alpha \alpha_{1} \alpha_{0}^{2} \beta \beta_{1} n^{2}+18 \alpha \alpha_{1}^{2} \beta \beta_{1}^{2} n^{2}=0 .
\end{aligned}
$$

For $\phi(\xi)^{1}$ :

$$
\begin{aligned}
& 2 a^{2} \alpha_{1} \alpha_{0} n^{2}+3 a_{2} \alpha_{1} \alpha_{0} \beta^{2} n-3 a_{1} \alpha_{1}^{2} \beta^{2}(n-1)+\frac{3}{2} a_{1} \alpha_{1}^{2} \beta^{2} n+6 a_{3} \alpha_{1} \beta^{2} \beta_{1}(n-1) \\
& +6 a_{3} \alpha_{1} \beta^{2} \beta_{1} n-6 \alpha_{1} \alpha_{0} \beta \nu n^{2}+12 \alpha \alpha_{1} \alpha_{0}^{3} \beta n^{2}+36 \alpha \alpha_{1}^{2} \alpha_{0} \beta \beta_{1} n^{2}=0 .
\end{aligned}
$$

For $\phi(\xi)^{2}$ :

$$
\begin{aligned}
& a^{2} \alpha_{1}^{2} n^{2}-3 a_{2} \alpha_{1}^{2} \beta^{2}(n-1)+3 a_{2} \alpha_{1}^{2} \beta^{2} n+\frac{9}{2} a_{3} \alpha_{0} \alpha_{1} \beta^{2} n+6 a_{4} \alpha_{1} \beta^{2} \beta_{1}(n-1) \\
& +6 a_{4} \alpha_{1} \beta^{2} \beta_{1} n-3 \alpha_{1}^{2} \beta \nu n^{2}+12 \alpha \alpha_{1}^{3} \beta \beta_{1} n^{2}+18 \alpha \alpha_{0}^{2} \alpha_{1}^{2} \beta n^{2}=0 .
\end{aligned}
$$

For $\phi(\xi)^{3}$ :

$$
-3 a_{3} \alpha_{1}^{2} \beta^{2}(n-1)+\frac{9}{2} a_{3} \alpha_{1}^{2} \beta^{2} n+6 a_{4} \alpha_{0} \alpha_{1} \beta^{2} n+12 \alpha \alpha_{0} \alpha_{1}^{3} \beta n^{2}=0 .
$$

For $\phi(\xi)^{4}$ :

$$
-3 a_{4} \alpha_{1}^{2} \beta^{2}(n-1)+6 a_{4} \alpha_{1}^{2} \beta^{2} n+3 \alpha \alpha_{1}^{4} \beta n^{2}=0 .
$$

Solving the system of equations (14-22) using Mathematica to get $\alpha_{0}, \alpha_{1}, \beta_{1}, a_{i} ; i=0,1, \ldots, 4$ and $\nu$. Thus, 
different types of solutions of equation (1) can be obtained as follows:

\section{Result 1:}

Setting $a_{0}=0, a_{1}=0$ and $a_{3}=0$, obtain:

$$
\alpha_{0}=0, \beta_{1}=0, a_{2}=\frac{3 \beta \nu n^{2}-a^{2} n^{2}}{3 \beta^{2}} \text { and } a_{4}=-\frac{\alpha \alpha_{1}^{2} n^{2}}{\beta(n+1)} .
$$

So, the corresponding solution of equation (1) can be written as:

$$
\begin{aligned}
& q(x, t)=3^{-\frac{1}{2 n}} \frac{\alpha_{1}}{\left|\alpha_{1}\right|} \times \\
& \left\{\sqrt{\frac{(n+1)\left(3 \beta \nu-a^{2}\right)}{\alpha \beta}} \operatorname{sech}\left((x-\nu t) \sqrt{\frac{3 \beta \nu n^{2}-a^{2} n^{2}}{3 \beta^{2}}}\right)\right\}^{1 / n} \times e^{i\left(\frac{a}{3 \beta} x-\frac{2 a^{3}}{27 \beta^{2}} t+\theta_{0}\right)},
\end{aligned}
$$

the solution represent a bright soliton with $3 \beta \nu-a^{2}>0$.

Or

$$
\begin{aligned}
& q(x, t)=3^{-\frac{1}{2 n}} \frac{\alpha_{1}}{\left|\alpha_{1}\right|} \times \\
& \left\{\sqrt{\frac{(n+1)\left(3 \beta \nu-a^{2}\right)}{\alpha \beta}} \sec \left((x-\nu t) \sqrt{\frac{3 \beta \nu n^{2}-a^{2} n^{2}}{3 \beta^{2}}}\right)\right\}^{1 / n} \times e^{i\left(\frac{a}{3 \beta} x-\frac{2 a^{3}}{27 \beta^{2}} t+\theta_{0}\right)},
\end{aligned}
$$

the solution represent a singular periodic wave solution with $3 \beta \nu-a^{2}>0$.

Result 2:

Setting $a_{0}=\frac{a_{2}^{2}}{4 a_{4}}, a_{1}=0$ and $a_{3}=0$, obtain:

$$
\alpha_{0}=0, \alpha_{1}=\frac{(n+1)\left(3 \beta \nu-a^{2}\right)}{12 \alpha \beta \beta_{1}}, a_{2}=-\frac{n^{2}\left(3 \beta \nu-a^{2}\right)}{6 \beta^{2}} \text { and } a_{4}=-\frac{n^{2}(n+1)\left(3 \beta \nu-a^{2}\right)^{2}}{144 \alpha \beta^{3} \beta_{1}^{2}} \text {. }
$$

So, the corresponding solution of equation (1) can be written as:

$$
\begin{aligned}
& q(x, t)=\frac{\left|\beta_{1}\right|}{\beta_{1}}\left\{\frac{(n+1)\left(3 \beta \nu-a^{2}\right) \sqrt{-\frac{\alpha \beta}{(n+1)\left(3 \beta \nu-a^{2}\right)}} \tanh \left(\frac{\sqrt{\frac{n^{2}\left(3 \beta \nu-a^{2}\right)}{3 \beta^{2}}}}{2}(x-\nu t)\right)}{2 \sqrt{3} \alpha \beta}+\right. \\
& \left.\frac{\operatorname{coth}\left(\frac{\sqrt{\frac{n^{2}\left(3 \beta \nu-a^{2}\right)}{3 \beta^{2}}}}{2}(x-\nu t)\right)}{2 \sqrt{3} \sqrt{-\frac{\alpha \beta}{(n+1)\left(3 \beta \nu-a^{2}\right)}}}\right\}^{1 / n} e^{i\left(\frac{a}{3 \beta} x-\frac{2 a^{3}}{27 \beta^{2}} t+\theta_{0}\right)}
\end{aligned}
$$

the solution represent a singular soliton with $3 \beta \nu-a^{2}>0$.

Or

$$
\begin{aligned}
& q(x, t)=\frac{\left|\beta_{1}\right|}{\beta_{1}}\left\{\frac{(n+1)\left(3 \beta \nu-a^{2}\right) \sqrt{-\frac{\alpha \beta}{(n+1)\left(3 \beta \nu-a^{2}\right)}} \tan \left(\frac{\sqrt{\frac{n^{2}\left(3 \beta \nu-a^{2}\right)}{3 \beta^{2}}}}{2}(x-\nu t)\right)}{2 \sqrt{3} \alpha \beta}+\right. \\
& \left.\frac{\cot \left(\frac{\sqrt{\frac{n^{2}\left(3 \beta \nu-a^{2}\right)}{3 \beta^{2}}}}{2}(x-\nu t)\right)}{2 \sqrt{3} \sqrt{-\frac{\alpha \beta}{(n+1)\left(3 \beta \nu-a^{2}\right)}}}\right\}^{1 / n} e^{i\left(\frac{a}{3 \beta} x-\frac{2 a^{3}}{27 \beta^{2}} t+\theta_{0}\right)}
\end{aligned}
$$


the solution represent a singular periodic wave solution with $3 \beta \nu-a^{2}<0$.

\section{Result 3:}

Setting $a_{0}=\frac{a_{2}^{2} m^{2}\left(1-m^{2}\right)}{a_{4}\left(2 m^{2}-1\right)^{2}}, a_{1}=0$ and $a_{3}=0$, obtain:

Case 1:

$$
\alpha_{0}=0, \alpha_{1}= \pm \frac{\sqrt{-a_{4} \beta-a_{4} \beta n}}{\sqrt{\alpha} n}, \beta_{1}=0, a_{2}=\frac{3 \beta \nu n^{2}-a^{2} n^{2}}{3 \beta^{2}} \text { and } m=1 .
$$

So, the corresponding solution of equation (1) can be written as:

$$
\left.q(x, t)= \pm 3^{-\frac{1}{2 n}}\left\{\sqrt{\frac{(n+1)\left(3 \beta \nu-a^{2}\right)}{\alpha \beta}} \operatorname{sech}\left(\sqrt{\frac{3 \beta \nu n^{2}-a^{2} n^{2}}{3 \beta^{2}}}(x-\nu t)\right)\right\}^{1 / n} \times e^{i\left(\frac{a}{3 \beta} x-\frac{2 a^{3}}{27 \beta^{2}} t+\theta_{0}\right.}\right)_{(30)}
$$

the solution represent a bright soliton with $3 \beta \nu-a^{2}>0$.

Case 2:

$$
\begin{aligned}
& \alpha_{0}=0, \alpha_{1}= \pm i \frac{\left(\frac{a^{2} \sqrt{a_{4}} \sqrt{\beta} \sqrt{n+1}}{\sqrt{\alpha}}-\frac{3 \sqrt{a_{4}} \beta^{3 / 2} \nu \sqrt{n+1}}{\sqrt{\alpha}}\right)}{a^{2} n-3 \beta \nu n}, \beta_{1}= \pm i \frac{n \sqrt{n+1}\left(a^{2}-3 \beta \nu\right)}{12 \sqrt{\alpha} \sqrt{a_{4}} \beta^{3 / 2}} \\
& a_{2}=-\frac{n^{2}\left(3 \beta \nu-a^{2}\right)}{6 \beta^{2}} \text { and } m=\frac{\sqrt{\sqrt{2}+2}}{2} .
\end{aligned}
$$

So, the corresponding solution of equation (1) can be written as:

$$
\begin{aligned}
& q(x, t)= \pm i\left\{\frac{\sqrt[4]{2} \sqrt{\frac{(n+1)\left(3 \beta \nu-a^{2}\right)}{\alpha \beta}} \mathrm{cn}\left(\frac{(x-t \nu) \sqrt{-\frac{n^{2}\left(3 \beta \nu-a^{2}\right)}{3 \beta^{2}}}}{\sqrt[4]{2}} \mid \frac{\sqrt{\sqrt{2}+2}}{2}\right)}{2 \sqrt{6-3 \sqrt{2}}}+\right.
\end{aligned}
$$

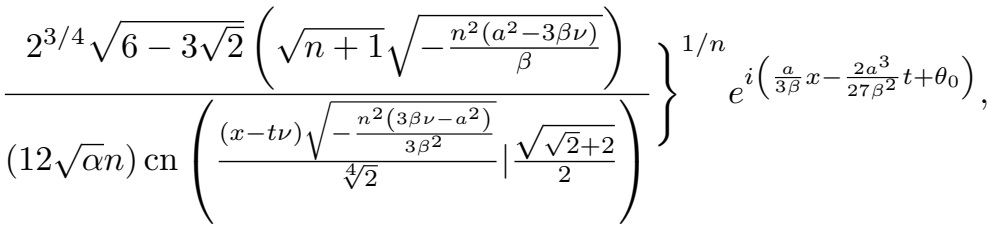

the solution represent a Jacobi elleptic function solution.

\section{Result 4:}

Setting $a_{0}=\frac{a_{2}^{2}\left(1-m^{2}\right)}{a_{4}\left(2-m^{2}\right)^{2}}, a_{1}=0$ and $a_{3}=0$, obtain:

$$
\alpha_{0}=0, \alpha_{1}= \pm \frac{\sqrt{-a_{4} \beta-a_{4} \beta n}}{\sqrt{\alpha} n}, \beta_{1}=0, a_{2}=\frac{3 \beta \nu n^{2}-a^{2} n^{2}}{3 \beta^{2}} \text { and } m=1 .
$$

So, the corresponding solution of equation (1) can be written as:

$$
q(x, t)=\left\{ \pm \frac{\sqrt{\beta+\beta n}}{\sqrt{\alpha} n} \operatorname{sech}\left(\frac{(x-\nu t) \sqrt{\frac{3 \beta \nu n^{2}-a^{2} n^{2}}{\beta^{2}}}}{\sqrt{3}}\right)\right\}^{1 / n} e^{i\left(\frac{a}{3 \beta} x-\frac{2 a^{3}}{27 \beta^{2}} t+\theta_{0}\right)}
$$


the solution represent a bright soliton solution with $3 \beta \nu-a^{2}>0$.

\section{Result 5:}

Setting $a_{0}=\frac{a_{2}^{2} m^{2}}{a_{4}\left(m^{2}+1\right)^{2}}, a_{1}=0$ and $a_{3}=0$, obtain:

$$
\begin{gathered}
\alpha_{0}=0, \alpha_{1}= \pm \frac{i\left(\frac{a^{2} \sqrt{a_{4}} \sqrt{\beta} \sqrt{n+1}}{\sqrt{\alpha}}-\frac{3 \sqrt{a_{4}} \beta^{3 / 2} \nu \sqrt{n+1}}{\sqrt{\alpha}}\right)}{a^{2} n-3 \beta \nu n}, \\
\beta_{1}= \pm \frac{i n \sqrt{n+1}\left(a^{2}-3 \beta \nu\right)}{12 \sqrt{\alpha} \sqrt{a_{4}} \beta^{3 / 2}}, a_{2}=-\frac{n^{2}\left(3 \beta \nu-a^{2}\right)}{6 \beta^{2}} \text { and } m=1 .
\end{gathered}
$$

So, the corresponding solution of equation (1) can be written as:

$$
\begin{aligned}
& q(x, t)= \pm \frac{i \sqrt{\beta} \sqrt{n+1} \sqrt{-\frac{n^{2}\left(a^{2}-3 \beta \nu\right)}{3 \alpha \beta^{2}}}}{2 n}\left\{\tanh \left(\frac{(x-\nu t) \sqrt{\frac{n^{2}\left(3 \beta \nu-a^{2}\right)}{\beta^{2}}}}{2 \sqrt{3}}\right)\right. \\
& \left.-\operatorname{coth}\left(\frac{(x-\nu t) \sqrt{\frac{n^{2}\left(3 \beta \nu-a^{2}\right)}{\beta^{2}}}}{2 \sqrt{3}}\right)\right\}^{1 / n} e^{i\left(\frac{a}{3 \beta} x-\frac{2 a^{3}}{27 \beta^{2}} t+\theta_{0}\right)},
\end{aligned}
$$

the solution can represent a singular soliton solution with $3 \beta \nu-a^{2}>0$.

\section{Result 6:}

Setting $a_{0}=0, a_{1}=0$ and $a_{4}=0$, obtain:

$$
\alpha_{1}=0, a_{2}=\frac{3 \beta \nu-a^{2}}{3 \beta^{2}}, a_{3}=\frac{2\left(3 \alpha_{0} \beta \nu-a^{2} \alpha_{0}\right)}{3 \beta^{2} \beta_{1}} \text { and } n=1 \text {, with } \alpha=0 \text {. }
$$

So, the corresponding solution of equation (1) can be written as:

$$
q(x, t)=\alpha_{0} \cosh \left(\frac{\sqrt{\beta \nu-\frac{a^{2}}{3}}(x-\nu t)}{\beta}\right) e^{i\left(\frac{a}{3 \beta} x-\frac{2 a^{3}}{27 \beta^{2}} t+\theta_{0}\right)},
$$

the solution represent a hyperbolic type solution with $3 \beta \nu-a^{2}>0$.

Or:

$$
q(x, t)=\alpha_{0} \cos \left(\frac{\sqrt{\beta \nu-\frac{a^{2}}{3}}(x-\nu t)}{\beta}\right) e^{i\left(\frac{a}{3 \beta} x-\frac{2 a^{3}}{27 \beta^{2}} t+\theta_{0}\right)},
$$

the solution represent a periodic wave solution with $3 \beta \nu-a^{2}>0$. 


\section{Result 7:}

Setting $a_{2}=0$ and $a_{4}=0$, obtain:

$$
\alpha_{1}=0, a_{0}=-\frac{\alpha \beta_{1}^{2}}{2 \beta}, a_{1}=-\frac{2 \alpha \alpha_{0} \beta_{1}}{\beta}, a_{3}=\frac{4 \alpha \alpha_{0}^{3}}{\beta \beta_{1}}, \nu=\frac{a^{2}+9 \alpha \alpha_{0}^{2} \beta}{3 \beta} \text { and } n=1 .
$$

So, the corresponding solution of equation (1) can be written as:

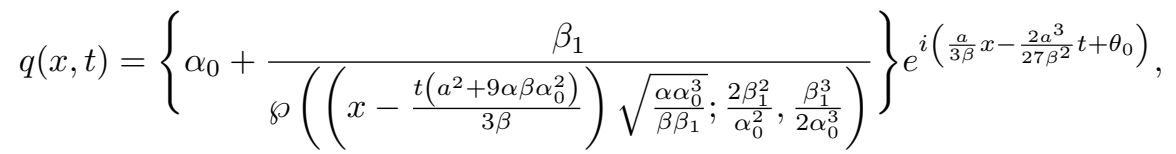

the solution represent a Weierstrass elliptic doubly periodic solution.

\section{Result 8:}

Setting $a_{0}=\frac{a_{1}^{2}}{4 a_{2}}, a_{3}=0$ and $a_{4}=0$, obtain:

$$
\alpha_{1}=0, \alpha_{0}= \pm \sqrt{\frac{3 \beta \nu-a^{2}}{3 \alpha \beta}}, a_{1}= \pm \frac{2 \sqrt{\alpha} \beta_{1} \sqrt{3 \beta \nu-a^{2}}}{\sqrt{3} \beta^{3 / 2}}, a_{2}=-\frac{2\left(3 \beta \nu-a^{2}\right)}{3 \beta^{2}}, \text { and } n=1 .
$$

So, the corresponding solution of equation (1) can be written as:

$$
q(x, t)=\left\{ \pm \frac{\sqrt{\frac{3 \beta \nu-a^{2}}{\alpha \beta}}}{\sqrt{3}}+\frac{\beta_{1}}{ \pm\left(\frac{3 \beta^{2}\left( \pm \frac{2 \sqrt{\alpha} \beta_{1} \sqrt{3 \beta \nu-a^{2}}}{\sqrt{\beta^{3} / 2}}\right)}{4\left(3 \beta \nu-a^{2}\right)}\right)+e^{ \pm \sqrt{\frac{2}{3}} \sqrt{-\frac{3 \beta \nu-a^{2}}{\beta^{2}}}(x-\nu t)}}\right\} e^{i\left(\frac{a}{3 \beta} x-\frac{2 a^{3}}{27 \beta^{2}} t+\theta_{0}\right)}
$$

Where $3 \beta \nu-a^{2}<0$.

\section{Result 9:}

Setting $a_{0}=0, a_{3}=0$ and $a_{4}=0$, obtain:

$$
\beta_{1}=0, a_{1}=\frac{2\left(3 \alpha_{0} \beta \nu-a^{2} \alpha_{0}\right)}{3 \alpha_{1} \beta^{2}}, a_{2}=\frac{3 \beta \nu-a^{2}}{3 \beta^{2}}, \alpha=0, \text { and } n=1 .
$$

So, the corresponding solution of equation (1) can be written as:

$$
q(x, t)=\left\{ \pm \alpha_{0} \sin \left(\frac{\sqrt{\frac{a^{2}-3 \beta \nu}{\beta^{2}}}(x-\nu t)}{\sqrt{3}}\right)\right\} e^{i\left(\frac{a}{3 \beta} x-\frac{2 a^{3}}{27 \beta^{2}} t+\theta_{0}\right)} .
$$

Where $3 \beta \nu-a^{2}<0$.

Or:

$$
q(x, t)=\left\{ \pm \alpha_{0} \sinh \left(\frac{2 \sqrt{-\frac{a^{2}-3 \beta \nu}{\beta^{2}}}(x-\nu t)}{\sqrt{3}}\right)\right\} e^{i\left(\frac{a}{3 \beta} x-\frac{2 a^{3}}{27 \beta^{2}} t+\theta_{0}\right)} .
$$


Where $3 \beta \nu-a^{2}>0$.

\section{Result 10:}

Setting $a_{1}=0, a_{3}=0$ and $a_{4}=0$, obtain:

$$
\alpha_{0}=0, \alpha_{1}=0, a_{0}=-\frac{\alpha \beta_{1}^{2} n^{2}}{\beta(n+1)}, \text { and } a_{2}=\frac{n^{2}\left(3 \beta \nu-a^{2}\right)}{3 \beta^{2}} .
$$

So, the corresponding solution of equation (1) can be written as:

$$
q(x, t)= \pm\left\{\frac{\csc \left(\frac{(x-\nu t) \sqrt{-\frac{n^{2}\left(3 \beta \nu-a^{2}\right)}{\beta^{2}}}}{\sqrt{3}}\right)}{\sqrt{3} \sqrt{\frac{\alpha \beta}{(n+1)\left(3 \beta \nu-a^{2}\right)}}}\right\}^{1 / n} e^{i\left(\frac{a}{3 \beta} x-\frac{2 a^{3}}{27 \beta^{2}} t+\theta_{0}\right)} .
$$

the solution represents a singular periodic solution with $3 \beta \nu-a^{2}<0$.

Or:

$$
q(x, t)= \pm\left\{\frac{\operatorname{csch}\left(\frac{(x-\nu t) \sqrt{-\frac{n^{2}\left(3 \beta \nu-a^{2}\right)}{\beta^{2}}}}{\sqrt{3}}\right)}{\left(\sqrt{3} \sqrt{-\frac{\alpha \beta}{(n+1)\left(3 \beta \nu-a^{2}\right)}}\right)}\right\}^{1 / n} e^{i\left(\frac{a}{3 \beta} x-\frac{2 a^{3}}{27 \beta^{2}} t+\theta_{0}\right)} .
$$

the solution represents a singular soliton with $3 \beta \nu-a^{2}<0$.

\section{Graphical representation of some solutions}

In this section, some of the the obtained solutions are represented graphically for certain values of $n, \beta, \alpha, \nu$ and $a$. Figure 1 shows $3 \mathrm{D}$ and $2 \mathrm{D}$ graphs of the bright soliton solution $(24)$ for $(n=0.5, \beta=1.34, \alpha=$ $2.88, \nu=0.66, a=0.7)$. Figure 2 shows $3 \mathrm{D}$ and $2 \mathrm{D}$ graphs of the singular periodic solution (25) for

$(n=0.5, \beta=3.08, \alpha=-2.3, \nu=1.88, a=2.72)$. Figure 3 shows $3 \mathrm{D}$ and $2 \mathrm{D}$ graphs of the singular soliton solution (27) for $(n=0.5, \beta=-1.42, \alpha=3.36, \nu=-2.36, a=2.24)$. Figure 4 shows 3D and 2D graphs of the singular periodic solution (28) for $(n=0.5, \beta=-1.36, \alpha=-0.66, \nu=-0.36, a=-2.74)$. Figure 5 shows $3 \mathrm{D}$ and $2 \mathrm{D}$ graphs of the bright soliton solution (30) for $(n=0.5, \beta=-2.42, \alpha=-1.42, \nu=$ $-0.52, a=0.76)$. Figure 6 shows $3 \mathrm{D}$ and $2 \mathrm{D}$ graphs of the singular periodic solution (48) for $(n=0.5, \beta=$ $0.82, \alpha=0.02, \nu=-0.4, a=-0.98)$. Figure 7 shows $3 \mathrm{D}$ and $2 \mathrm{D}$ graphs of the singular soliton solution (49) for $(n=0.5, \beta=-2.04, \alpha=-1.52, \nu=-0.82, a=-2.26)$. 


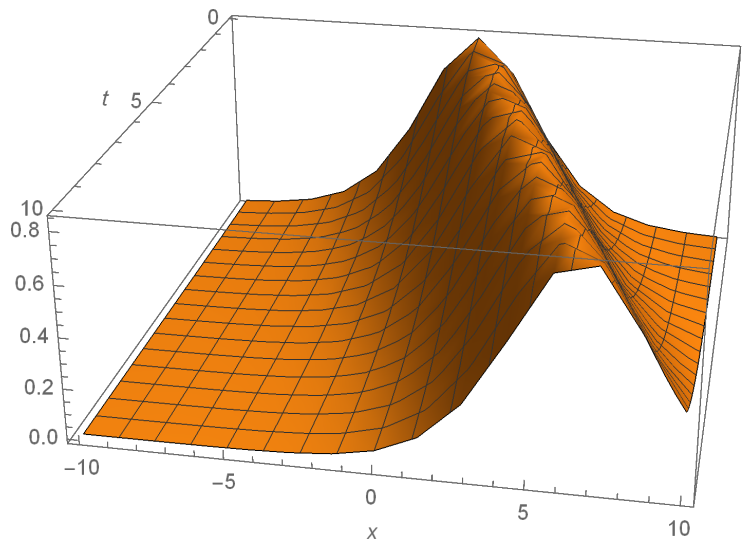

(a)

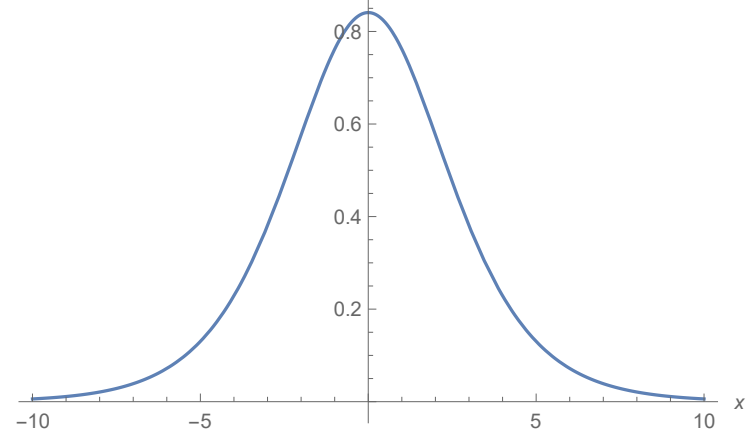

(b)

Figure 1: 3D and 2D graphs of the bright soliton solution (24) for $(n=0.5, \beta=1.34, \alpha=2.88, \nu=0.66, a=$ $0.7)$.

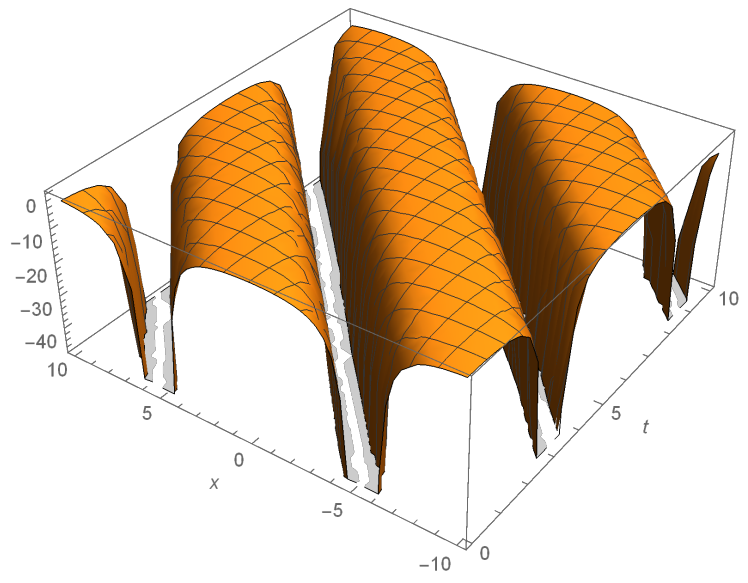

(a)

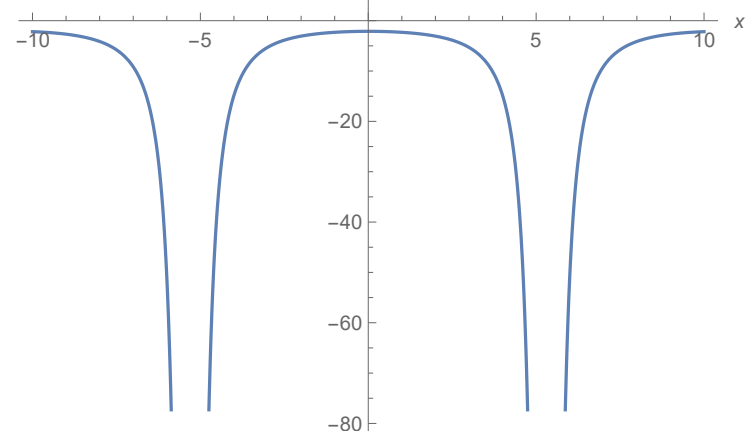

(b)

Figure 2: 3D and 2D graphs of the singular periodic solution (25) for $(n=0.5, \beta=3.08, \alpha=-2.3, \nu=$ $1.88, a=2.72)$. 


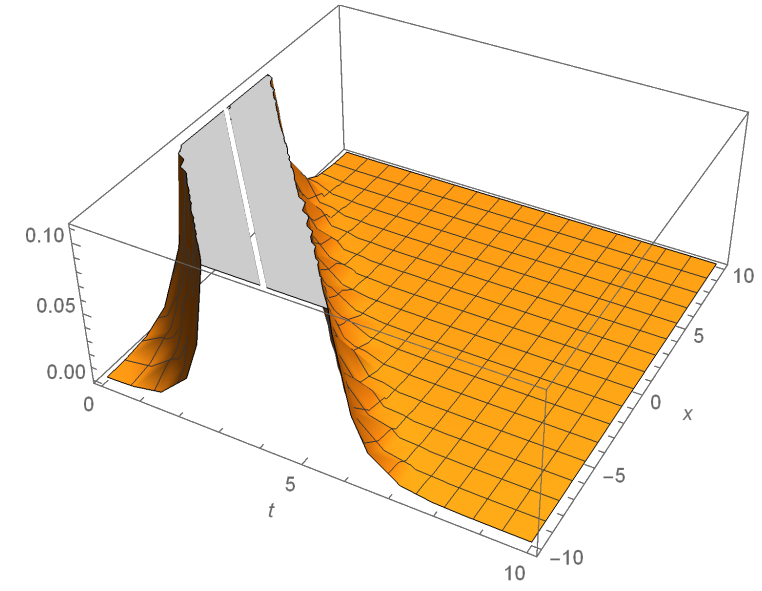

(a)

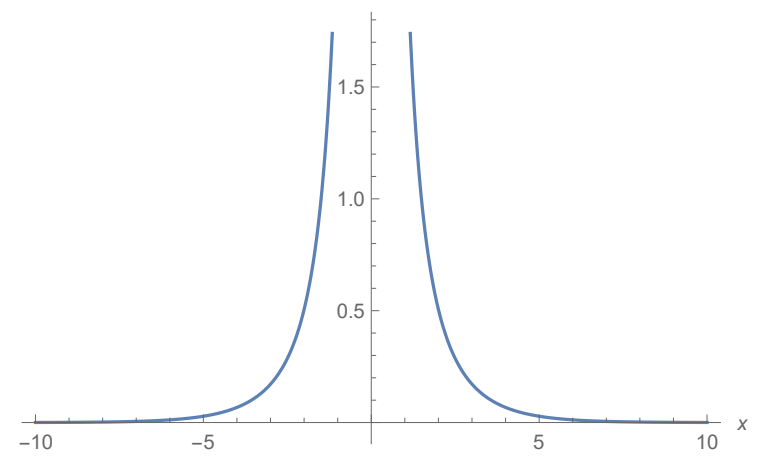

(b)

Figure 3: 3D and 2D graphs of the singular soliton solution (27) for $(n=0.5, \beta=-1.42, \alpha=3.36, \nu=$ $-2.36, a=2.24)$.

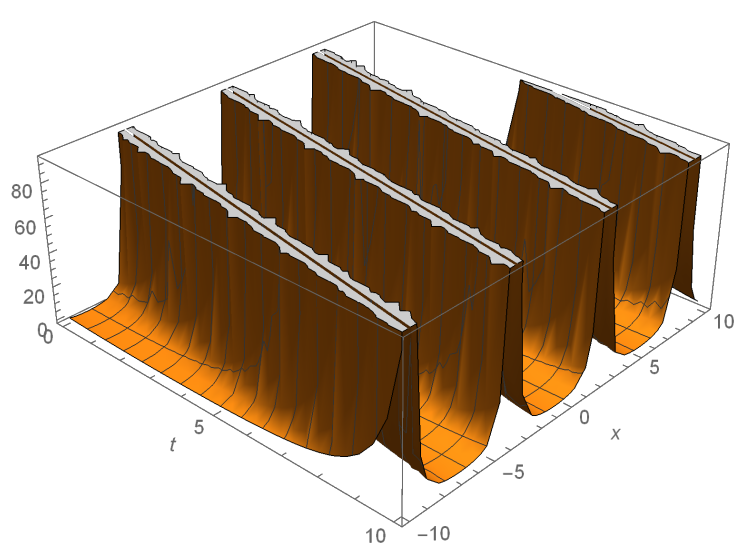

(a)

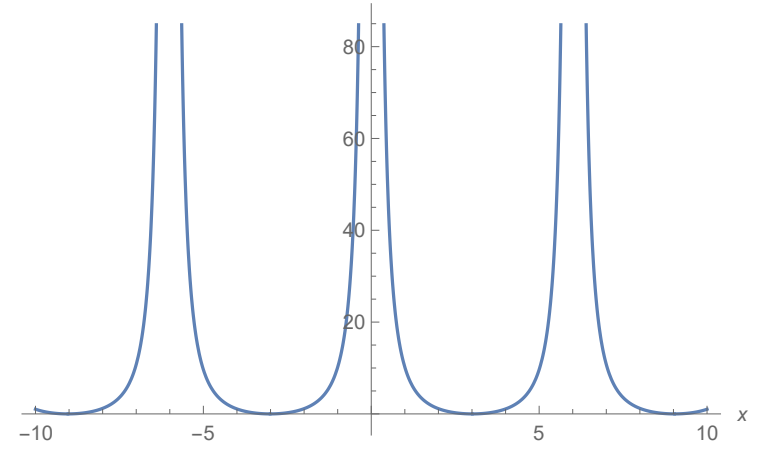

(b)

Figure 4: 3D and 2D graphs of the singular periodic solution (28) for $(n=0.5, \beta=-1.36, \alpha=-0.66, \nu=$ $-0.36, a=-2.74)$. 


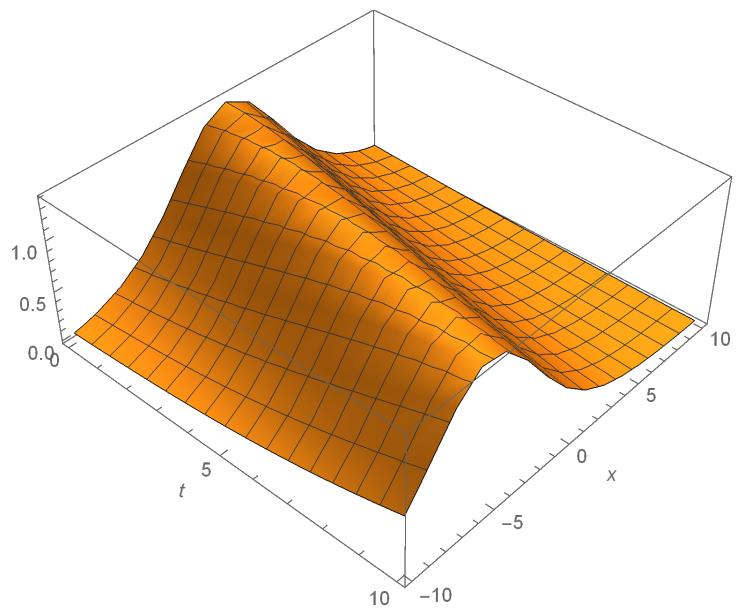

(a)

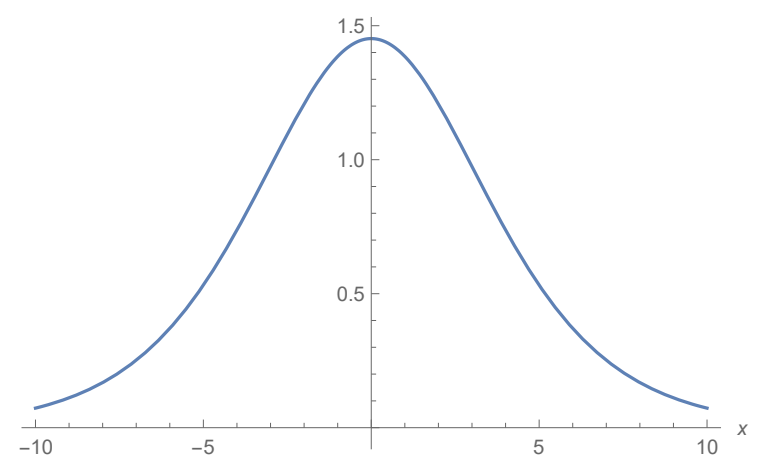

(b)

Figure 5: 3D and 2D graphs of the bright soliton solution (30) for $(n=0.5, \beta=-2.42, \alpha=-1.42, \nu=$ $-0.52, a=0.76)$.

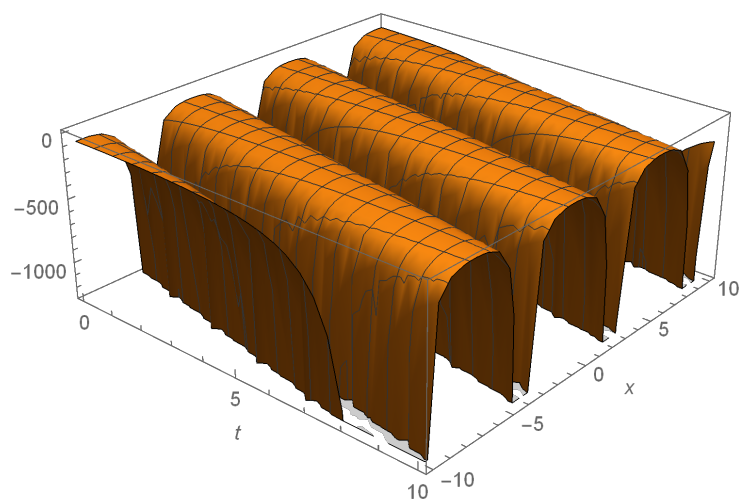

(a)

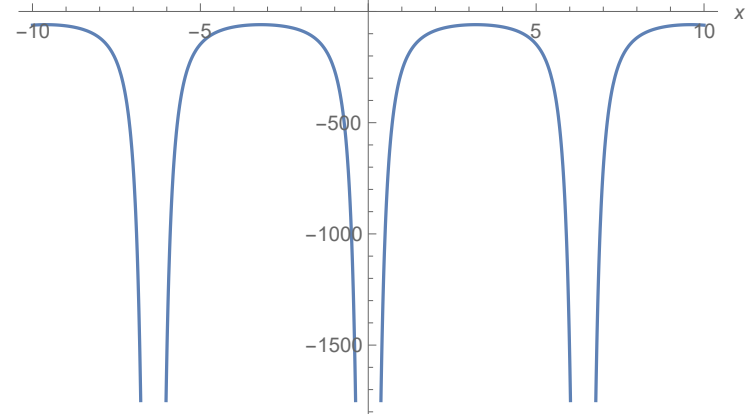

(b)

Figure 6: 3D and 2D graphs of the singular periodic solution (48) for $(n=0.5, \beta=0.82, \alpha=0.02, \nu=$ $-0.4, a=-0.98)$. 


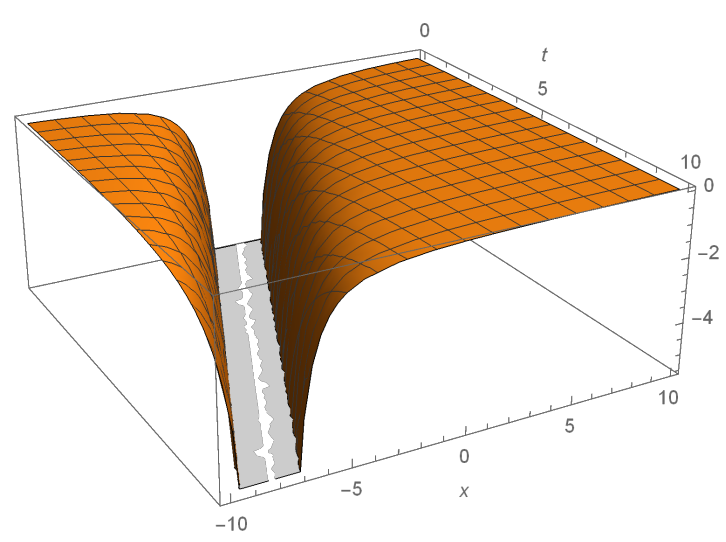

(a)

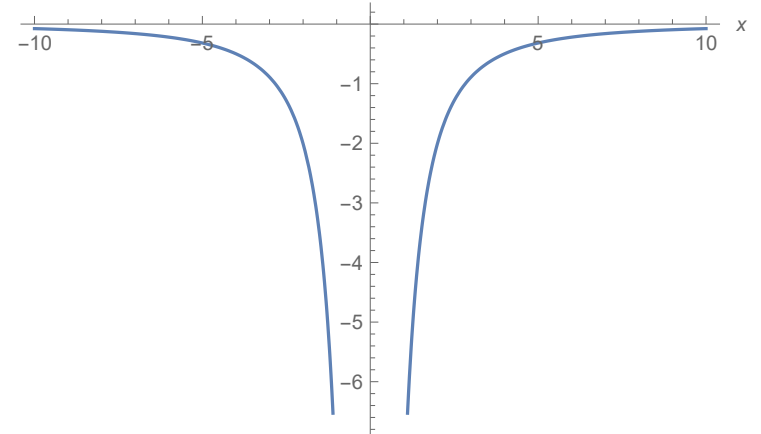

(b)

Figure 7: 3D and 2D graphs of the singular soliton solution (49) for $(n=0.5, \beta=-2.04, \alpha=-1.52, \nu=$ $-0.82, a=-2.26)$.

\section{Conclusion}

In this article, the Radhakrishnan-Kundu-Laksmannan equation that describes the wave propagation in optical fibers been studied successfully by applying the improved modified extended tanh-function method. Various types of solutions were extracted such as bright solitons, singular solitons, singular periodic wave solutions, Jacobi elliptic solutions, periodic wave solutions and Weierstrass elliptic doubly periodic solutions. Moreover, 3D and 2D plots of some solutions were introduced to show their features.

\section{Competing interests}

The authors declare that they have no competing interests.

\section{Authors' contributions}

The authors declare that the study was realized in collaboration with the same responsibility. All authors read and approved the manuscript.

\section{Author details}

${ }^{1}$ Department of Physics and Mathematics Engineering, Faculty of Engineering,

Ain Shams University, Cairo, Egypt.

${ }^{2}$ Department of Physics and Engineering Mathematics, Higher Institute of Engineering, El-Shorouk Academy, El-Shorouk City, Cairo, Egypt.

\section{References}

[1] A. Biswas, 1-soliton solution of the generalized Radhakrishnan, Kundu, Lakshmanan equation, Physics Letters A 373(2009) 2546-2548.

[2] Jianming Zhang, Shuming Li, Hongpeng Geng, Bifurcations of exact travelling wave solutions for the generalized RKL equation, J. Appl. Anal. Comput.. 6(2016) 1205-1210.

[3] Anupma Bansal, Anjan Biswas, Mohammad F Mahmood, Qin Zhou, Mohammad Mirzazadeh, Ali Saleh Alshomrani, Seithuti P Moshokoa, and Milivoj Belic, , Optical soliton perturbation with Radhakrishnan-Kundu-Lakshmanan equation by Lie group analysis, Optik 163(2018) 137-141. 
[4] Anjan Biswas, Mehmet Ekici, Abdullah Sonmezoglu, and Ali Saleh Alshomrani, Optical solitons with Radhakrishnan-Kundu-Lakshmanan equation by extended trial function scheme, Optik 160(2018) 415427 .

[5] Anjan Biswas, Optical soliton perturbation with Radhakrishnan-Kundu-Lakshmanan equation by traveling wave hypothesis, Optik. 171(2018) 217-220.

[6] Nikolay A Kudryashov, Dariya V Safonova, and Anjan Biswas, Painlevé Analysis and a Solution to the Traveling Wave Reduction of the Radhakrishnan-Kundu-Lakshmanan Equation, Regular and Chaotic Dynamics 24(2019) 607-614.

[7] Nikolay A Kudryashov, The Radhakrishnan-Kundu-Lakshmanan equation with arbitrary refractive index and its exact solutions, Optik 238(2021) 166738.

[8] M. M. A. El-Sheikh, H. M. Ahmed, A. H Arnous, W. B. Rabie, A. Biswas, S. Khan, A. S. Alshomrani \& M. R. Belic, Optical solitons with differential group delay for coupled Kundu-Eckhaus equation using extended simplest equation approach, Optik 208(2020) 164051.

[9] N. A. Kudryashov, A generalized model for description of propagation pulses in optical fiber, Optik 189(2019), 42-52.

[10] N. A. Kudryashov, Mathematical model of propagation pulse in optical fiber with power nonlinearities, Optik 212(2020) 164750.

[11] N. A. Kudryashov, First integrals and general solution of the Fokas-Lenells equation, Optik 195(2019), 163135.

[12] N. Mahak \& G. Akram, The modified auxiliary equation method to investigate solutions of the perturbed nonlinear Schrödinger equation with Kerr law nonlinearity, Optik 207(2020) 164467.

[13] S. T. R. Rizvi, K. Ali \& M. Ahmad, Optical solitons for Biswas-Milovic equation by new extended auxiliary equation method, 204(2020) 164181.

[14] M. Tahir \& A. U. Awan, Optical singular and dark solitons with Biswas-Arshed model by modified simple equation method, Optik 202(2020) 163523.

[15] X-B. Wang, T-T. Zhang, M-J. Dong, Dynamics of the breathers and rogue waves in the higher-order nonlinear Schrödinger equation, Applied Mathematics Letters 86(2018) 298-304.

[16] A-M. Wazwaz. Partial Differential Equations and Solitary Waves Theory. Springer Verlag, New York, NY. USA. (2009).

[17] Adel Darwish, Aly R. Seadawy, Hamdy M. Ahmed, A. L. Elbably, Mohammed F. Shehab, and Ahmed H. Arnous, Study on soliton solutions of the longitudinal wave equation and magneto-electro-elastic circular rod dynamical model, International Journal of Modern Physics B 35, (2021), 2150168.

[18] Y. Yıldırım, A. Biswas, S. Khan, A. S. Alshomrani \& M. R Belic, Optical solitons with differential group delay for complex Ginzburg-Landau equation having Kerr and parabolic laws of refractive index, Optik 202(2020) 163737.

[19] Y. Yıldırım, Optical solitons to Kundu-Mukherjee-Naskar model with modified simple equation approach, Optik 184(2019) 247-252. 
[20] Y. Yıldırım, A. Biswas, M. Ekici, E. M. E. Zayed, A. K. Alzahrani \& M. R. Belic, Optical soliton perturbation, with maximum intensity, having generalized Kudryashov's law of refractive index, Optik 227(2021) 165328.

[21] E. M. E. Zayed, M. E. M. Alngar, M. El-Horbaty, A. Biswas, A. S. Alshomrani, S. Khan, M. Ekici \& H. Triki, Optical solitons in fiber Bragg gratings having Kerr law of refractive index with extended Kudryashov's method and new extended auxiliary equation approach, Chinese Journal of Physics 66(2020) 187-205.

[22] Hamdy M. Ahmed, Wafaa B. Rabie, Structure of optical solitons in magneto-optic waveguides with dual-power law nonlinearity using modified extended direct algebraic method, Optical and Quantum Electronics 53, (2021), 1-26.

[23] H. Rezazadeh, A. Korkmaz, M. M. Khater, M. Eslami, D. Lu, and R. A. Attia. New exact traveling wave solutions of biological population model via the extended rational sinh-cosh method and the modified Khater method, Modern Physics Letters B 33, (2019), 1950338.

[24] Islam Samir, Niveen Badra, Aly R. Seadawy, Hamdy M. Ahmed, Exact wave solutions of the fourth order non-linear partial differential equation of optical fiber pulses by using different methods, Optik, 230 (2021), 166313.

[25] Aly R. Seadawy, Hamdy M. Ahmed, Wafaa B. Rabie, and Anjan Biswas, An alternate pathway to solitons in magneto-optic waveguides with triple-power law nonlinearity, Optik 231 (2021), 166480.

[26] E. M. E. Zayed, A-G. Al-Nowehy, M. E. M. Alngar, A. Biswas, M. Asma, M. Ekici, A. K. Alzahrani \& M. R. Belic, Highly dispersive optical solitons in birefringent fibers with four nonlinear forms using Kudryashov's approach, Optik(2021) 1-12.

[27] Taher A Nofal, Elsayed ME Zayed, Mohamed EM Alngar, Reham MA Shohib, and Mehmet Ekici, Highly dispersive optical solitons perturbation having Kudryashov's arbitrary form with sextic-power law refractive index and generalized non-local laws, Optik 228(2020) 166120.

[28] Z. Yang, B.Y.C. Hon, An improved modified extended tanh function method, Z. Naturforsch. 61 (2006) $103-115$. 